\title{
Human hydrocephalus dynamics: loss of normal pulsation absorber mechanism
} Eun-Hyoung Park*1, Per K Eide ${ }^{2}$ and Joseph R Madsen ${ }^{1}$

\author{
Address: ${ }^{1}$ Department of Neurosurgery, Children's Hospital Boston, Harvard Medical School, 300 Longwood Avenue, Boston, MA 02115, USA \\ and 2Department of Neurosurgery, The National Hospital (Rikshospitalet), Sognvannsvn. 20, N-0027 Oslo, Norway \\ Email: Eun-Hyoung Park* - eun-hyoung.park@childrens.harvard.edu \\ * Corresponding author
}

from 52nd Annual Meeting of the Society for Research into Hydrocephalus and Spina Bifida Providence, RI, USA. II-I 4 June 2008

Published: 3 February 2009

Cerebrospinal Fluid Research 2009, 6(SuppI I):S28 doi:I0.1 186/1743-8454-6-SI-S28

This abstract is available from: http://www.cerebrospinalfluidresearch.com/content/6/SI/S28

(C) 2009 Park et al; licensee BioMed Central Ltd.

\section{Background}

We have studied ten patient undergoing evaluations of presumed normal pressure hydrocephalus (NPH) based on the result of systems analysis with data obtained from canines developing hydrocephalus which have shown evidence of alterations in the notch filter-like intracranial pulsation absorber. All human patients tested had clinical evidence of hydrocephalus, prompting evaluation of the canine condition, suggesting that the notch filter mechanism, which may malfunction in canine hydrocephalus.

\section{Materials and methods}

We hypothesize that a notch filter mechanism can also be found in the human intracranial system and if so, the mismatch of notch frequency relative to the cardiac frequency increases, and performance efficacy of the pulsation absorber decreases in hydrocephalic patients. To test the hypothesis, we have applied the time-varying transfer function (TVTF) method using arterial blood pressure (ABP) input and intracranial pressure (ICP) output obtained from the patients. ICP pulse wave amplitude has also been used to examine its relationship to the notch filter performance.

\section{Results}

The preliminary results show that 1 ) notch filter appears to be present in the human brain; 2) the tendency towards relatively large frequency mismatch between the notch frequency and the cardiac frequency shown in hydrocephalic canines has also been observed in all patients studied. On average, the largest mismatch was around 1.5 $\mathrm{Hz}$; and 3) for most of the cases, elevated ICP pulse wave amplitude appears to be directly related to low efficacious performance of the notch filter.

\section{Conclusion}

The results suggest that a notch filter (pulsation absorber) can be found in the human intracranial system, and that notch frequency in the pathological condition appears to be tuned to the frequencies other than the cardiac frequency or fluctuates around the cardiac frequency, which is similar to that shown in hydrocephalic canines. Pathophysiological alterations with NPH in humans resemble those seen in chronic canine models of hydrocephalus. These findings may also suggest diagnostic and therapeutic approaches in normal pressure hydrocephalus and a role for the notch filter in normal cerebral blood flow. 